\title{
THEORETICAL PREDICTIONS FOR SCATTERING FROM ELASTIC SOLID CYLINDERS WITH HEMISPHERICAL CAPS AND COMPARISON WITH DATA
}

\author{
M.F. WERBY and C.E. DEAN \\ Naval Oceanographic and Atmospheric Research Laboratory, Numerical Modeling Division, Building \\ 1100, Stennis Space Center, MS 39529-5004, USA
}

\begin{abstract}
The extended boundary condition equations due to Waterman have led to a T-matrix that can describe scattering from axially symmetric targets over a broad frequency range. Recent interest in scattering from cylinders with hemispherical end caps has prompted us to analyze several examples from Le Havre. All phenomena predicted by the T-matrix calculations are confirmed by the data. In addition, bending resonances are predicted by the theoretical calculations and compared with predictions from bar theory. A series of predictions are made for various materials and aspect ratios to aid future experimental work.
\end{abstract}

Les équations des conditions étendues aux limites de Waterman nous conduit à une matrice de transfert qui peut décrire la diffusion par des cibles à symétrie axiale pour un grand spectre de fréquences. L'intérêt récent pour la diffusion par les cylindres aux bouts hémisphériques nous incite à l'analyse de plusieurs exemples provenant du Havre. Tous les phénomènes prédits par les calculs de matrices de transfert se confirment par les données. De plus, les résonances de flexion se prédisent par les calculs théoriques et se comparent aux prédictions de la théorie des barres. Une série de prédictions se fait pour des matières diverses et pour des rapports divers de longueur à largeur de la cible pour aider les travaux expérimentaux futurs.

\section{Introduction}

Back scattered echoes yield interesting information when scattering from elastic targets, particularly in the resonance region. Generally one has source and receiver at the same location for a fixed orientation. If one adjusts the orientation over a suitable angular region then it is possible to ascertain certain symmetries of the target if they exist particularly if one varies the frequencies over a suitable range of resonances. If one observes axial symmetry then it is possible to obtain both the dimensions of the object and the aspect ratio of the object (ratio of length to width). Once this information is obtained it is also possible to obtain information on whether the target is a solid or a shell and in some cases what the shell thickness is as well as the material properties of the target. This is assuming that the target is in a "free" environment; that is, the boundaries of the target are not a factor in calculation. Time domain responses for specific pulse types also yield information, and it is easy to see how a series of questions can form the basis of a scenario that can rule out certain targets or lead to a probability (confidence level) that specific targets are present.

Some interest has arisen for the case of scattering from elastic solid cylinders with hemispherical endcaps. We have performed a considerable number of calculations for spheroids with aspect ratios out to 10 for six materials. In this work we will focus on scattering from a tungsten carbide (WC) cylinder with hemispherical end-caps and an aspect ratio of 2 . We will describe calculations for scattering end-on, broadside, and at $30^{\circ}, 45^{\circ}$, and $60^{\circ}$ relative to the axis of symmetry. A brief discussion of the methodology and physical notions that pertain to the final analysis is presented in the next section with the final section containing the analysis. 


\section{Formal expressions}

The theoretical calculations presented here are performed using normal mode theory for the spherical cases and by the extended boundary condition (EBC) or T-matrix method for the nonspherical cases. The EBC (T-Matrix) methods have been published elsewhere so we will not present the details here. The boundary conditions and pertinent points are, however, indicated below. The boundary conditions at a fluid-elastic interface are

$$
n \cdot U_{+}=n \cdot U_{-}, \quad n \cdot t_{+}=n \cdot t, \quad n \times t=0,
$$

where $n, U$, and $t$ are the outward unit normal to the object, the displacement vector, and the traction on the surface of the scatterer, respectively. The $+(-)$ sign indicates an exterior (interior) point relative to the elastic object. The T-matrix relates the known partial wave components $\left\{A_{n}\right\}$ of the incident field (here a plane wave) with the unknown partial wave components $\left\{f_{n}\right\}$ of the scattered field as follows:

$$
\mathrm{f}_{\mathrm{n}}=\Sigma \mathrm{T}_{\mathrm{nn}} \cdot A_{\mathrm{n}}
$$

The T-matrix, which is derived from the extended boundary condition method, is symmetric and for nonlossy objects obeys the generalized optical theorem. These factors can be built into the formalism or $T$ can be checked to assure that all the properties are satisfied, and that convergence has been achieved. The form function is obtained from the T-matrix and the incident field from the expression:

$$
f_{\infty}(\theta)=2(k L / 2)\left|\Sigma\left[(2 n+1)\left(2 n^{\prime}+1\right)\right]^{1 / 2} i^{n}-n^{\prime} T_{n n^{\prime}} P_{n^{\prime}}(\cos \theta) A_{n}\right| .
$$

The rationale behind the standing-wave argument for determining resonances is based on the fact that for special cases one can equate the measure $P$ around the perimeter of the object (in the direction of the circumnavigating field) with a half-integral number of wavelengths. For the case (here) for elastic solids we are close to a rigid background and one has

$$
P=\lambda_{p}(N+1 / 2),
$$

where $\mathbf{N}=2,3,4, \ldots$ By conservation of frequency one can show that

$$
\lambda_{\mathrm{p}}=\lambda_{\mathrm{w}}\left(\mathrm{V}_{\mathrm{p}} / \mathrm{V}_{\mathrm{w}}\right)
$$

Since $k L / 2=2 \pi L / \lambda_{w}$, this leads to the standing wave condition for a resonance

$$
[\mathrm{kL} / 2]_{\operatorname{Res}}=\mathrm{L} \pi\left(\mathrm{V}_{\mathrm{p}} / \mathrm{V}_{\mathrm{w}}\right)(\mathrm{N}+1 / 2) / \mathrm{P}
$$

Überall, et al. have shown that broadside resonances for cylinders can be obtained from the simple expression

$$
[k L / 2]_{\text {Res }}=(L / D)\left(V_{p} / V_{w}\right)(N+1 / 2)
$$

Here, we may use the phase velocity for the spherical case in place of $\mathrm{V}_{\mathrm{p}}$. Thus with simple expressions one can predict resonance locations for both end-on and broadside resonances. At oblique incidence one can not only excite surface resonances but also bending resonances. It has been shown that those resonances are related to bending or flexural modes which are also predictable using Timoshenko theory for a finite circular cylinder (which approximates a spheroid). The expression for the resonance location in that case is as follows:

$$
[\mathrm{kL} / 2]_{\operatorname{Res}}=\pi^{2} / 8 \sqrt{2}(\mathrm{Cl} / \mathrm{Cw})(2 \mathrm{n}-1)^{2} /\left[\alpha^{2}+\left(\alpha^{2}+3 \Gamma \pi^{2}(\mathrm{n}-1)^{2} / 8\right)^{1 / 2}\right],
$$

where $\alpha$ designates the aspect ratio and $\Gamma$ is a material parameter. 


\section{Analysis of results}

We briefly analyse the results for scattering from a tungsten carbide cylinder with hemispherical endcaps for a range of dimensionless half length size parameter $\mathrm{kL} / 2=0$ to 20 where $\mathrm{L}$ is the length of the object and $k$ the wave number of the incident field. Often one chooses $r$, the radius of the target, in place of the half length $\mathrm{L} / 2$; in that case $\mathrm{kr}$ would range from 0 to 10 . We examine results, end-on, broadside, and at oblique angles of incidence. In the future, we will examine results out to $\mathrm{kL} / 2=30$, and we will subtract a rigidbackground from the form function leaving only the resonance response. It is to be remembered that we expect to observe Rayleigh and whispering gallery resonances for both end-on and broadside incidence.

Fig. 1 illustrates the backscattered case for end-on incidence. One observes the familiar Franz or creeping wave pattern out to about $k L / 2=8$ where a strong $(2,1)$ Rayleigh resonance is present. One also observes the $(3,1)$ and $(4,1)$ resonances resonances at about $\mathrm{kL} / 2=14$ and 19 , respectively. Whispering gallery resonances are observed at intermediate values. Scattering at oblique angles relative to the axis of symmetry yields a more complicated pattern. This is to be expected because at oblique angles resonances can be excited corresponding to standing waves circumnavigating the largest geodesic (a meridian) and the shortest (parallel to the equator of the target). Further, flexural or bending modes can also be excited at oblique angles. Fig. 2 illustrates the case for backscattering with broadside incidence. There we see a weak Franz wave effect characteristic of cylinders as well as a bending mode at about $\mathrm{kL} / 2=5.5$ and the end-on Rayleigh resonance at about $\mathrm{kL} / 2=8$. If we analyze these results by analogy with backscattering from spheroids then the first major dip at about $\mathrm{kL} / 2=13.5$ is the first broadside Rayleigh resonance while the second dip just to the right of the $(2,1)$ resonance is the $(2,2)$ end-on whispering gallery resonance followed by two broadside whispering gallery resonances and finally the $(3,1)$ broadside resonance at about $\mathrm{kL} / 2=20$. Fig. 3 has the same features as the broadside scattering case including the end-on resonances but with an additional effect caused by bending modes. The lowest bending mode $(\mathrm{n}=$ 2) occurrs at about $k L / 2=2.75$ and the second mode $(n=3)$ at about $k L / 2=5.5$. They are manifested as sharp peaks and are less pronounced for $n>3$. It has been shown that these resonances are predicted using Timoshenko beam theory. Figs. 4 and 5 correspond to scattering at $30^{\circ}$ and $60^{\circ}$ relative to the axis of symmetry. All features are present in these cases with the bending mode being strongest for the $60^{\circ}$ case.

\section{Summary}

These calculations are part of on-going research on scattering from axially symmetric targets. Extended calculations are to follow in whichthe background will be subtracted to manifest the resonances more clearly. It is felt that these results coincide with recently published experimental results from Le Havre.

\section{Bibliography}

[1] P. C. Waterman, "Matrix theory of elastic wave scattering," J. Acoust. Soc. Am. 60, 567 (1976).

[2] A. Bostrom, "Scattering of stationary acoustic waves by an elastic obstacle immersed in a fluid," $J$. Acoust. Soc. Am. 67, 390-398 (1982).

[3] M. F. Werby and G. J. Tango, J. Acoust. Soc. Am. 77, 2081 (1986). 


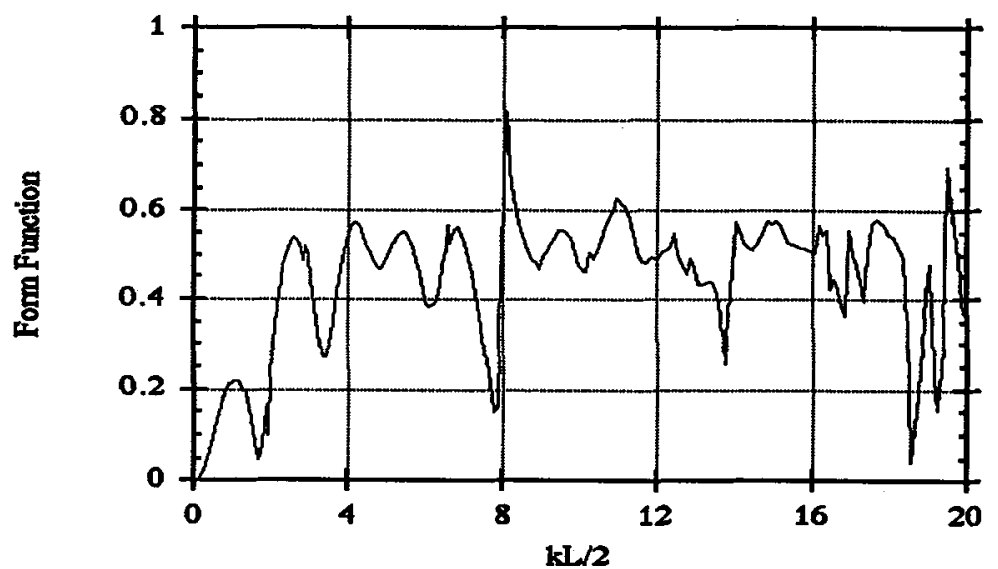

Fig. 1. Backscattered form function vs. kL/2 for end-on incidence.

[Réponse en fréquence de rétrodiffusion pour incidence selon l'axe de symétrie.]

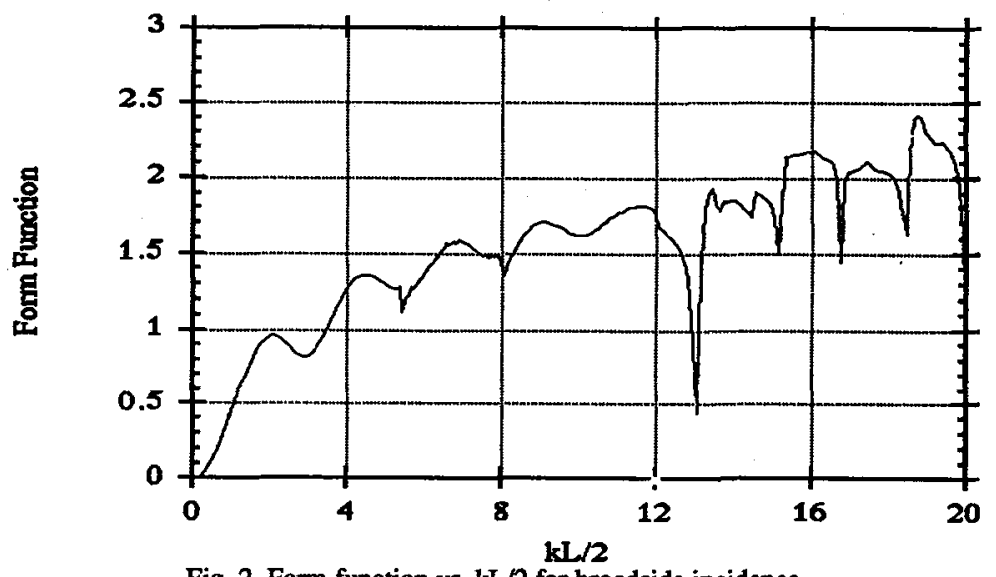

Fig. 2. Form function vs. kL/2 for broadside incidence.

[Réponse en fréquence de rétrodiffusion pour incidence à $90^{\circ}$ de l'axe de symétrie.]

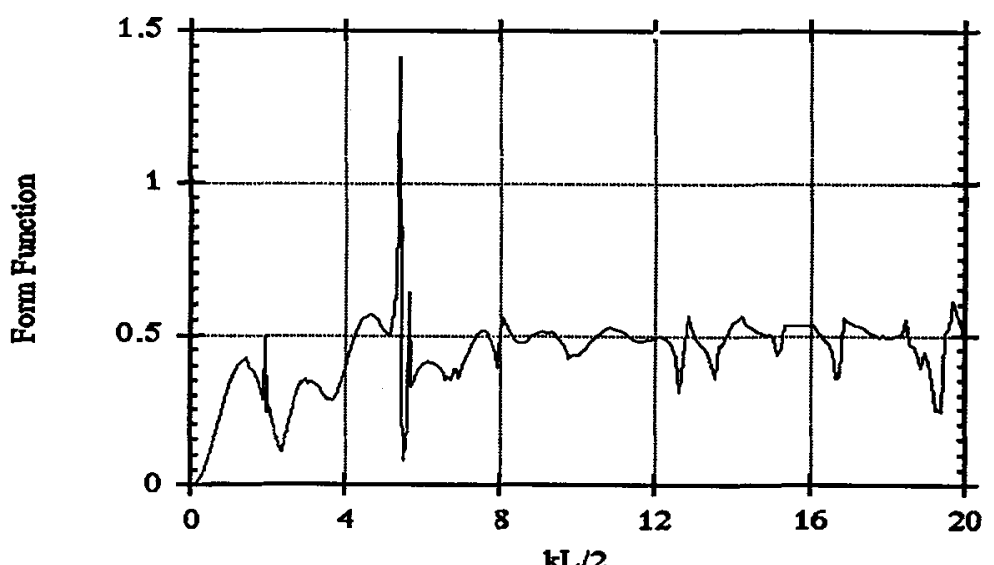

Fig. 3. Form function vs. $\mathrm{kL} / 2$ for incidence at $45^{\circ}$ to the axis of symmetrie. [Réponse en fréquence de rétrodiffusion pour incidence à $45^{\circ}$ de l'axe de symétrie.] 